Homology, Homotopy and Applications, vol.10(2), 2008, pp.211-226

\title{
A UNIVERSALITY THEOREM FOR VOEVODSKY'S ALGEBRAIC COBORDISM SPECTRUM
}

\author{
IVAN PANIN, KONSTANTIN PIMENOV AND OLIVER RÖNDIGS
}

(communicated by J. F. Jardine)

\begin{abstract}
An algebraic version of a theorem of Quillen is proved. More precisely, for a regular Noetherian scheme $S$ of finite Krull dimension, we consider the motivic stable homotopy category $\mathrm{SH}(S)$ of $\mathbf{P}^{1}$-spectra, equipped with the symmetric monoidal structure described in $[\mathbf{7}]$. The algebraic cobordism $\mathbf{P}^{1}$-spectrum MGL is considered as a commutative monoid equipped with a canonical orientation $t h^{\mathrm{MGL}} \in \mathrm{MGL}^{2,1}(\mathrm{Th}(\mathcal{O}(-1)))$. For a commutative monoid $E$ in the category $\mathrm{SH}(S)$, it is proved that the assignment $\varphi \mapsto \varphi\left(t h^{\mathrm{MGL}}\right)$ identifies the set of monoid homomorphisms $\varphi$ : MGL $\rightarrow E$ in the motivic stable homotopy category $\mathrm{SH}(S)$ with the set of all orientations of $E$. This result generalizes a result of G. Vezzosi in [12].
\end{abstract}

\section{Introduction}

Quillen proved in $[\mathbf{1 0}]$ that the formal group law associated to the complex cobordism spectrum MU is the universal one on the Lazard ring. As a consequence, the set of orientations on a commutative ring spectrum $E$ in the stable homotopy category is in bijective correspondence with the set of homomorphisms of ring spectra from MU to $E$ in the stable homotopy category. This result allowed a whole new approach to understanding the stable homotopy category, which is still actively pursued today.

On the algebraic side of things, there is a similar $\mathbf{P}^{1}$-ring spectrum MGL in the motivic stable homotopy category of a Noetherian finite-dimensional scheme $S$. The formal group law associated to MGL is not known to be the universal one, although unpublished work of Hopkins and Morel claims this if $S$ is the spectrum of a field of characteristic zero. Nevertheless, the set of orientations on a $\mathbf{P}^{1}$-ring spectrum in the motivic stable homotopy category over $S$ can be identified in the same fashion if $S$ is regular.

Theorem 1.1. Let $S$ be a regular Noetherian finite-dimensional scheme, and let $E$ be a commutative $\mathbf{P}^{1}$-ring spectrum over $S$. The set of orientations on $E$ is in bijection with the set of homomorphisms of $\mathbf{P}^{1}$-ring spectra from MGL to $E$ in the motivic stable homotopy category over $S$.

Received January 4, 2008, revised July 17, 2008; published on November 14, 2008. 2000 Mathematics Subject Classification: 14F05, 55N22, 55P43.

Key words and phrases: algebraic cobordism, motivic ring spectra.

This article is available at http://intlpress.com/HHA/v10/n2/a11

Copyright (C) 2008, International Press. Permission to copy for private use granted. 
For a more detailed formulation, see Theorem 2.7. Our main motivation to write this paper was to prove the universality theorem 1.1 in a form convenient for its application in [8]. Theorem 1.1 has already been employed in $[\mathbf{1}]$ and $[\mathbf{1 1}]$. In the special case where $S$ is the spectrum of a field, Theorem 1.1 was stated originally in a slightly different form by G. Vezzosi in [12], although he ignored certain aspects of the multiplicative structure on MGL.

\subsection{Preliminaries}

We refer to [7, Appendix] for the basic terminology, notation, constructions, definitions and results. For the convenience of the reader we recall the basic definitions. Let $S$ be a regular Noetherian scheme of finite Krull dimension. One may think of $S$ being the spectrum of a field or the integers. Below we need to apply [5, Prop. 4.3.8], which is the basic reason to work with a regular base scheme $S$. Let $\mathcal{S} m / S$ be the category of smooth quasi-projective $S$-schemes, and let sSet be the category of simplicial sets. A motivic space over $S$ is a functor

$$
A: S m / S^{\text {op }} \rightarrow \text { sSet }
$$

(see [7, A.1.1]). The category of motivic spaces over $S$ is denoted $\mathbf{M}(S)$. This definition of a motivic space is different from the one considered by Morel and Voevodsky in [5]; they consider only those simplicial presheaves which are sheaves in the Nisnevich topology on $S m / S$. With our definition, the Thomason-Trobaugh $K$-theory functor obtained by using big vector bundles is a motivic space on the nose. It is not a simplicial Nisnevich sheaf. This is why we prefer to work with the above notion of "space".

We write $\mathrm{H}_{\bullet}^{\mathrm{cm}}(S)$ for the pointed motivic homotopy category and $\mathrm{SH}^{\mathrm{cm}}(S)$ for the stable motivic homotopy category over $S$ as constructed in [7, A.3.9, A.5.6]. By [7, A.3.11, resp. A.5.6] there are canonical equivalences to $\mathrm{H}_{\bullet}(S)$ of [5], respectively, $\mathrm{SH}(S)$ of $[\mathbf{1 3}]$. Both $\mathrm{H}_{\bullet}^{\mathrm{cm}}(S)$ and $\mathrm{SH}_{\bullet}^{\mathrm{cm}}(S)$ are equipped with closed symmetric monoidal structures such that the $\mathbf{P}^{1}$-suspension spectrum functor is a strict symmetric monoidal functor

$$
\Sigma_{\mathbf{P}^{1}}^{\infty}: \mathrm{H}_{\bullet}^{\mathrm{cm}}(S) \rightarrow \mathrm{SH}^{\mathrm{cm}}(S)
$$

Here $\mathbf{P}^{1}$ is considered as a motivic space pointed by $\infty \in \mathbf{P}^{1}$. The symmetric monoidal structure $\left(\wedge, \mathbb{I}_{S}=\Sigma_{\mathbf{P}^{1}}^{\infty} S_{+}\right)$on the homotopy category $\mathrm{SH}^{\mathrm{cm}}(S)$ is constructed on the model category level by employing symmetric $\mathbf{P}^{1}$-spectra. It satisfies the properties required by Theorem 5.6 of Voevodsky's talk $[\mathbf{1 3}]$. From now on we will usually omit the superscript $(-)^{\mathrm{cm}}$.

Every $\mathbf{P}^{1}$-spectrum $E=\left(E_{0}, E_{1}, \ldots\right)$ represents a cohomology theory on the category of pointed motivic spaces. Namely, for a pointed motivic space $(A, a)$ set

$$
E^{p, q}(A, a)=\operatorname{Hom}_{\mathrm{SH}}(S)\left(\Sigma_{\mathbf{P}^{1}}^{\infty}(A, a), \Sigma^{p, q}(E)\right)
$$

and $E^{*, *}(A, a)=\oplus_{p, q} E^{p, q}(A, a)$. This definition extends to motivic spaces via the functor $A \mapsto A_{+}$which adds a disjoint basepoint. That is, for a non-pointed motivic space $A$, set $E^{p, q}(A)=E^{p, q}\left(A_{+},+\right)$and $E^{*, *}(A)=\oplus_{p, q} E^{p, q}(A)$. Recall that there is a canonical element in $E^{2 n, n}\left(E_{n}\right)$, denoted as $\Sigma_{\mathbf{P}^{1}}^{\infty} E_{n}(-n) \rightarrow E$. It is represented by the canonical map $\left(*, \ldots, *, E_{n}, E_{n} \wedge \mathbf{P}^{1}, \ldots\right) \rightarrow\left(E_{0}, E_{1}, \ldots, E_{n}, \ldots\right)$ of $\mathbf{P}^{1}$-spectra. 
Every $X \in \mathcal{S} m / S$ defines a representable motivic space constant in the simplicial direction, taking an $S$-smooth scheme $U$ to $\operatorname{Hom}_{S m / S}(U, X)$. It is not possible in general to choose a basepoint for representable motivic spaces. So we regard $S$-smooth varieties as motivic spaces (non-pointed) and set

$$
E^{p, q}(X)=E^{p, q}\left(X_{+},+\right)
$$

Given a $\mathbf{P}^{1}$-spectrum $E$ we will reduce the double grading on the cohomology theory $E^{*, *}$ to a grading. Namely, set $E^{m}=\oplus_{m=p-2 q} E^{p, q}$ and $E^{*}=\oplus_{m} E^{m}$.

To complete this section, we define a $\mathbf{P}^{1}$-ring spectrum to be a monoid $(E, \mu, e)$ in $\left(\mathrm{SH}(\mathrm{S}), \wedge, \mathbb{I}_{S}\right)$. A commutative $\mathbf{P}^{1}$-ring spectrum is a commutative monoid $(E, \mu, e)$ in $\left(\mathrm{SH}(\mathrm{S}), \wedge, \mathbb{I}_{S}\right)$. The cohomology theory $E^{*}$ defined by a $\mathbf{P}^{1}$-ring spectrum is a ring cohomology theory. The cohomology theory $E^{*}$ defined by a commutative $\mathbf{P}^{1}$-ring spectrum is a ring cohomology theory, however it is not necessarily graded commutative. The cohomology theory $E^{*}$ defined by an oriented commutative $\mathbf{P}^{1}$-ring spectrum is a graded commutative ring cohomology theory, as will be explained in Subsection 1.3.

\subsection{Oriented commutative ring spectra}

Following Adams and Morel, we define an orientation of a commutative $\mathbf{P}^{1}$-ring spectrum. However we prefer to use Thom classes instead of Chern classes. Consider the pointed motivic space $\mathbf{P}^{\infty}=\operatorname{colim}_{n \geqslant 0} \mathbf{P}^{n}$ having basepoint $g_{1}: S=\mathbf{P}^{0} \hookrightarrow \mathbf{P}^{\infty}$.

The tautological "vector bundle" $\mathcal{T}(1)=\mathcal{O}_{\mathbf{P}}(-1)$ is also known as the Hopf bundle. It has zero section $z: \mathbf{P}^{\infty} \hookrightarrow \mathcal{T}(1)$. The fiber over the point $g_{1} \in \mathbf{P}^{\infty}$ is $\mathbb{A}^{1}$. For a vector bundle $V$ over a smooth $S$-scheme $X$, with zero section $z: X \hookrightarrow V$, its Thom space $\operatorname{Th}(V)$ is the Nisnevich sheaf associated to the presheaf

$$
Y \mapsto V(Y) /(V \backslash z(X))(Y)
$$

on the Nisnevich site $\mathcal{S} m / S$. In particular, $\operatorname{Th}(V)$ is a pointed motivic space in the sense of [7, Defn. A.1.1]. It coincides with Voevodsky's Thom space [13, p. 422], since $\operatorname{Th}(V)$ already is a Nisnevich sheaf. The Thom space of the Hopf bundle is then defined as the colimit $\operatorname{Th}(\mathcal{T}(1))=\operatorname{colim}_{n \geqslant 0} \operatorname{Th}\left(\mathcal{O}_{\mathbf{P}^{n}}(-1)\right)$. Abbreviate $T=\operatorname{Th}\left(\mathbf{A}_{S}^{1}\right)$.

Let $E$ be a commutative $\mathbf{P}^{1}$-ring spectrum. The unit gives rise to an element $1 \in E^{0,0}(S)$. Applying the $\mathbf{P}^{1}$-suspension isomorphism to that element we get an element $\Sigma_{\mathbf{P}^{1}}(1) \in E^{2,1}\left(\mathbf{P}^{1}, \infty\right)$. The canonical covering of $\mathbf{P}^{1}$ defines motivic weak equivalences

$$
\mathbf{P}^{1} \stackrel{\sim}{\longrightarrow} \mathbf{P}^{1} / \mathbf{A}^{1} \stackrel{\sim}{\longleftarrow} \mathbf{A}^{1} / \mathbf{A}^{1} \backslash\{0\}=T
$$

of pointed motivic spaces inducing isomorphisms

$$
E\left(\mathbf{P}^{1}, \infty\right) \leftarrow E\left(\mathbf{A}^{1} / \mathbf{A}^{1} \backslash\{0\}\right) \rightarrow E(T) .
$$

Let $\Sigma_{T}(1)$ be the image of $\Sigma_{\mathbf{P}^{1}}(1)$ in $E^{2,1}(T)$.

Definition 1.2. Let $E$ be a commutative $\mathbf{P}^{1}$-ring spectrum. A Thom orientation of $E$ is an element $t h \in E^{2,1}(\operatorname{Th}(\mathcal{T}(1))$ such that its restriction to the Thom space of the fibre over the distinguished point coincides with the element $\Sigma_{T}(1) \in E^{2,1}(T)$. A Chern orientation of $E$ is an element $c \in E^{2,1}\left(\mathbf{P}^{\infty}\right)$ such that $\left.c\right|_{\mathbf{P}^{1}}=-\Sigma_{\mathbf{P}^{1}}(1)$. An orientation of $E$ is either a Thom orientation or a Chern orientation. One says 
that a Thom orientation th of $E$ coincides with a Chern orientation $c$ of $E$ provided that $c=z^{*}(t h)$, or equivalently the element th coincides with $t h(\mathcal{O}(-1))$ given by $(3)$ below.

Remark 1.3. The element th should be regarded as the Thom class of the tautological line bundle $\mathcal{T}(1)=\mathcal{O}(-1)$ over $\mathbf{P}^{\infty}$. The element $c$ should be regarded as the Chern class of the tautological line bundle $\mathcal{T}(1)=\mathcal{O}(-1)$ over $\mathbf{P}^{\infty}$.

Example 1.4. The following orientations given below are relevant for our work. Here MGL denotes the $\mathbf{P}^{1}$-ring spectrum representing algebraic cobordism obtained below in Definition 2.4, and BGL denotes the $\mathbf{P}^{1}$-ring spectrum representing algebraic $K$-theory constructed in [7, Theorem 2.2.1].

- Let $u_{1}: \Sigma_{\mathbf{P}^{1}}^{\infty} \operatorname{Th}(\mathcal{T}(1))(-1) \rightarrow$ MGL be the canonical map of $\mathbf{P}^{1}$-spectra. Set $t h^{\mathrm{MGL}}=u_{1} \in \mathrm{MGL}^{2,1}(\mathrm{Th}(\mathcal{T}(1)))$. Since the equality

$$
\left.t h^{\mathrm{MGL}}\right|_{\operatorname{Th}(\mathbf{1})}=\Sigma_{T}(1)
$$

holds in $\mathrm{MGL}^{2,1}(\mathrm{Th}(\mathbf{1}))$, the class $t h^{\mathrm{MGL}}$ is an orientation of MGL.

- Set $c^{K}=(-\beta) \cup([\mathcal{O}]-[\mathcal{O}(1)]) \in \mathrm{BGL}^{2,1}\left(\mathbf{P}^{\infty}\right)$. The relation (11) from $[\mathbf{7}]$ shows that the class $c^{K}$ is an orientation of BGL.

\subsection{Certain properties of oriented $\mathbf{P}^{1}$-ring spectra}

Let $E$ be a commutative $\mathbf{P}^{1}$-ring spectrum and $E^{*, *}$ the cohomology theory it represents. For an element $\lambda \in \Gamma\left(S, \mathcal{O}_{S}^{*}\right)$, denote by $\Lambda$ the morphism $\mathbf{P}^{1} \rightarrow \mathbf{P}^{1}$ which maps $[x: y]$ to $[x: \lambda y]$. Let $\Lambda^{*}: E^{*, *}\left(\mathbf{P}^{1}, \infty\right) \rightarrow E^{*, *}\left(\mathbf{P}^{1}, \infty\right)$ be the pull-back map induced by $\Lambda$. Let $\Sigma_{\mathbf{P}^{1}}: E^{*, *}(S) \rightarrow E^{*+2, *+1}\left(\mathbf{P}^{1}, \infty\right)$ be the suspension isomorphism. Set

$$
\epsilon=\left(\Sigma_{\mathbf{P}^{1}}^{-1} \circ(-1)^{*} \circ \Sigma_{\mathbf{P}^{1}}\right)(1) \in E^{0,0}(S) .
$$

Clearly $\epsilon^{2}=1$. The following commuting rule is proved by Morel in [4]: for any $a \in E^{p, q}$ and $b \in E^{r, s}$ one has $a \cup b=(-1)^{p s} \epsilon^{q r} b \cup a$. Suppose that $\epsilon=1$ for $E$. Define a Chern structure on $E^{*, *}$ as an assignment which associates to every $X \in \mathcal{S} m(S)$ and every line bundle $L$ over $X$ a class $c(L) \in E^{2,1}(X)$ such that

(1) the class $c(L)$ is natural,

(2) $c(\mathbf{1})=0$ (the class of a trivial bundle vanishes), and

(3) the set $\{1, c(\mathcal{O}(-1))\}$ is a basis of the two-sided $E^{*, *}(S)$-module $E^{*, *}\left(\mathbf{P}^{1} \times S\right)$.

Given a Chern structure on $E^{*, *}$, one can state and prove the projective bundle theorem, construct a theory of Chern classes, and construct a theory of Thom classes by repeating literally the arguments and constructions from [6, Thm. 3.9, Thm. 3.27 and Proof of Thm. 3.35]. The resulting theory of Chern classes is uniquely defined by the property that for line bundles the classes $c_{1}$ and $c$ coincide. The resulting theory of Thom classes is uniquely defined by the property that for every line bundle $L$ with zero section $z$ one has $z^{*}(t h(L))=c(L)$. We recall the construction of the theory of Thom classes at the end of this section. 
Below, in this section, $(E, t h)$ is an oriented commutative ring $\mathbf{P}^{1}$-spectrum. The class $c=z^{*}(t h) \in E^{2,1}\left(\mathbf{P}^{\infty}\right)$ is a Chern orientation of $E$ by [9, Prop. 6.5.1]. Clearly the pull-back map $E^{*, *}\left(\mathbf{P}^{2}\right) \rightarrow E^{*, *}\left(\mathbf{P}^{1}\right)$ is surjective. We claim now that for any $\lambda \in \Gamma\left(S, \mathcal{O}_{S}^{*}\right)$ one has $\Lambda^{*}=\mathrm{id}$. In fact, to check this just repeat the arguments from $[\mathbf{2}$, Proof of Lemma 1.6]. So $\epsilon=1$ if $E$ is orientable.

Now one can produce a Chern structure on $E^{*, *}$ as follows. The scheme $S$ is regular. The functor isomorphism $\operatorname{Hom}_{\mathrm{H}_{\bullet}(S)}\left(-, \mathbf{P}^{\infty}\right) \rightarrow \operatorname{Pic}(-)$ on the category $\mathcal{S} m / S$, provided by [5, Thm. 4.3.8], sends the class of the identity map $\mathbf{P}^{\infty} \rightarrow \mathbf{P}^{\infty}$ to the class of the tautological line bundle $\mathcal{O}(-1)$ over $\mathbf{P}^{\infty}$. For a line bundle $L$ over

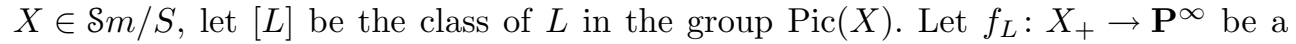
morphism in $\mathrm{H}_{\bullet}(S)$ corresponding to the class $[L]$ under the functor isomorphism above. For a line bundle $L$ over $X \in \mathcal{S} m / S$, set $c(L)=f_{L}^{*}(c) \in E^{2,1}(X)$. Clearly, $c(\mathcal{O}(-1))=c$. The assignment $L / X \mapsto c(L)$ is a Chern structure on $\left.E^{*, *}\right|_{\delta m \mathcal{O} p}$ since $\left.c\right|_{\mathbf{P}^{1}}=-\Sigma_{\mathbf{P}^{1}}(1) \in E^{2,1}\left(\mathbf{P}^{1}, \infty\right)$. With that Chern structure, $\left.E^{*, *}\right|_{\delta m \mathcal{O} p}$ is an oriented ring cohomology theory in the sense of $[6]$. In particular, $\left(\mathrm{BGL}, c^{K}\right)$ defines an oriented ring cohomology theory on $\mathcal{S} m \mathcal{O} p$.

Combining the results given above, we obtain a theory of Thom classes

$$
V \mapsto t h(V) \in E^{2 \operatorname{rank}(V), \operatorname{rank}(V)}(\operatorname{Th}(V))
$$

on $E^{*, *}$. The latter means that the classes $t h(V)$ are natural, multiplicative, and satisfy the Thom isomorphism property.

Theorem 1.5. For a rank $r$ vector bundle $p: V \rightarrow X$ on $X \in \mathcal{S} m / S$ with zero section $z: X \hookrightarrow V$, the map

$$
-\cup \operatorname{th}(V): E^{*, *}(X) \rightarrow E^{*+2 r, *+r}(\operatorname{Th}(V))
$$

is an isomorphism of two-sided $E^{*, *}(X)$-modules, where $-\cup t h(V)$ is written for the composition map $(-\cup \operatorname{th}(V)) \circ p^{*}$.

Additionally we have a normalization property: $\operatorname{th}(\mathbf{1})=\Sigma_{T}(1) \in E^{2,1}(\operatorname{Th}(\mathbf{1}))$ as one can see from the relations (2) and (3) below. In fact,

$$
\overline{t h}(\mathbf{1})=c\left(\mathcal{O}_{\mathbf{P}^{1}}(1)\right)=-c\left(\mathcal{O}_{\mathbf{P}^{1}}(-1)\right)=\Sigma_{\mathbf{P}^{1}}(1) .
$$

(The second relation here holds by [6, Lemma 3.6].) Thus $t h(\mathbf{1})=\Sigma_{T}(1)$.

Analogous to [13, p. 422], one obtains for vector bundles $V \rightarrow X$ and $W \rightarrow Y$ in $\mathcal{S} m / S$ a canonical map of pointed motivic spaces $\operatorname{Th}(V) \wedge \operatorname{Th}(W) \rightarrow \operatorname{Th}\left(V \times{ }_{S} W\right)$, which is a motivic weak equivalence as defined in [7, Defn. 3.1.6]. In fact, the canonical map becomes an isomorphism after Nisnevich (even Zariski) sheafification. In the special case where $Y=S$ and $W=\mathbf{1}$ is the trivial line bundle, this motivic weak equivalence has the form $\operatorname{Th}(V) \wedge T \rightarrow T h(V \oplus \mathbf{1})$.

Corollary 1.6. For $W=V \oplus \mathbf{1}$ consider the composite motivic weak equivalence

$$
\omega: \operatorname{Th}(V) \wedge \mathbf{P}^{1} \rightarrow \operatorname{Th}(V) \wedge \mathbf{P}^{1} / \mathbf{A}^{1} \leftarrow \operatorname{Th}(V) \wedge T \rightarrow \operatorname{Th}(W)
$$


of pointed motivic spaces over $S$ (see diagram (1) on page 213). The diagram

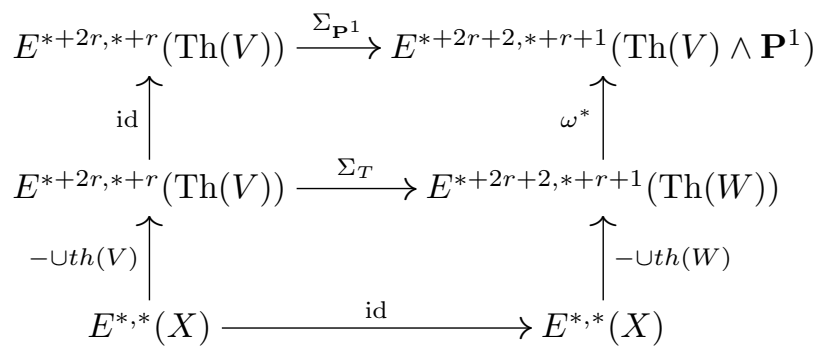

commutes.

Proof. The bottom square in this diagram commutes by the multiplicativity of Thom classes and the normalization property of the class th(1). The top one commutes by definition.

We conclude this section by recalling briefly how the associated theory of Thom classes is constructed. Given the Chern structure above, there is a unique theory of Chern classes $V \mapsto c_{i}(V) \in E^{2 i, i}(X)$ such that for every line bundle $L$ on $X$ one has $c_{1}(L)=c(L)$. For a rank $r$ vector bundle $V$ over $X$ consider the vector bundle $W:=\mathbf{1} \oplus V$ and the associated projective space bundle $\mathbf{P}(W)$ of lines in $W$. Set

$$
\bar{t} h(V)=c_{r}\left(p^{*}(V) \otimes \mathcal{O}_{\mathbf{P}(W)}(1)\right) \in E^{2 r, r}(\mathbf{P}(W)) .
$$

It follows from [6, Cor. 3.18] that the support extension map

$$
E^{2 r, r}(\mathbf{P}(W) /(\mathbf{P}(W) \backslash \mathbf{P}(\mathbf{1}))) \rightarrow E^{2 r, r}(\mathbf{P}(W))
$$

is injective and $\bar{t} h(E) \in E^{2 r, r}(\mathbf{P}(W) /(\mathbf{P}(W) \backslash \mathbf{P}(\mathbf{1})))$. Set

$$
\operatorname{th}(E)=j^{*}(\bar{t} h(E)) \in E^{2 r, r}\left(\mathrm{Th}_{X}(V)\right),
$$

where $j: \operatorname{Th}_{X}(V) \rightarrow \mathbf{P}(W) /(\mathbf{P}(W) \backslash \mathbf{P}(\mathbf{1}))$ is the canonical motivic weak equivalence of pointed motivic spaces induced by the open embedding $V \hookrightarrow \mathbf{P}(W)$. The assignment $V / X$ to $t h(V)$ is a theory of Thom classes on $E^{*, *}$ (see the proof of [6, Thm. 3.35]). Moreover $t h(\mathcal{O}(-1))=$ th in $E^{2,1}\left(\mathbf{P}^{\infty}\right)$.

\section{Cohomology of infinite Grassmannians}

Let $\mathbf{G r}(n, n+m)$ be the Grassmann scheme of $n$-dimensional linear subspaces of $\mathbf{A}_{S}^{n+m}$. The closed embedding $\mathbf{A}_{S}^{n+m}=\mathbf{A}_{S}^{n+m} \times\{0\} \hookrightarrow \mathbf{A}_{S}^{n+m+1}$ defines a closed embedding

$$
\operatorname{Gr}(n, n+m) \hookrightarrow \operatorname{Gr}(n, n+m+1) .
$$

The tautological vector bundle is denoted $\mathcal{T}(n, n+m) \rightarrow \mathbf{G r}(n, n+m)$. The closed embedding (4) is covered by a map $\mathcal{T}(n, n+m) \hookrightarrow \mathcal{T}(n, n+m+1)$ of vector bundles. Let $\mathbf{G r}(n)=\operatorname{colim}_{m \geqslant 0} \mathbf{G r}(n, n+m), \mathcal{T}(n)=\operatorname{colim}_{m \geqslant 0} \mathcal{T}(n, n+m)$ and $\operatorname{Th}(\mathcal{T}(n))=$ $\operatorname{colim}_{m \geqslant 0} \operatorname{Th}(\mathcal{T}(n, n+m))$. These colimits are taken in the category of motivic spaces over $S$. 
Remark 2.1. It is not difficult to prove that $E^{*, *}(\mathbf{G r}(n, n+m))$ is multiplicatively generated by the Chern classes $c_{i}(\mathcal{T}(n, n+m))$ of the vector bundle $\mathcal{T}(n, n+m)$. This proves the surjectivity of the map $E^{*, *}(\mathbf{G r}(n, n+m+1)) \rightarrow E^{*, *}(\mathbf{G r}(n, n+m))$ and

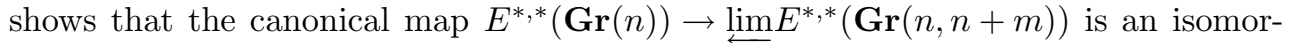
phism. Thus for each $i$ there exists a unique element $c_{i}=c_{i}(\mathcal{T}(n)) \in E^{2 i, i}(\mathbf{G r}(n))$, which for each $m$ restricts to the element $c_{i}(\mathcal{T}(n, n+m))$ under the obvious pull-back map.

Theorem 2.2. Let $(E, c)$ be an oriented commutative $\mathbf{P}^{1}$-ring spectrum. Then

$$
E^{*, *}(\mathbf{G r}(n))=E^{*, *}(S)\left[\left[c_{1}, c_{2}, \ldots, c_{n}\right]\right]
$$

is the formal power series ring, where $c_{i}:=c_{i}(\mathcal{T}(n)) \in E^{2 i, i}(\mathbf{G r}(n))$ denotes the $i$-th Chern class of the tautological bundle $\mathcal{T}(n)$. The inclusion $\operatorname{inc}_{n}: \mathbf{G r}(n) \hookrightarrow \mathbf{G r}(n+1)$ satisfies $\operatorname{inc}_{n}^{*}\left(c_{m}\right)=c_{m}$ for $m<n+1$ and $\operatorname{inc}_{n}^{*}\left(c_{n+1}\right)=0$.

Proof. The case $n=1$ is well-known (see for instance [6, Thm. 3.9]). For a finitedimensional vector space $W$ and a positive integer $m$, let $\mathbf{F}(m, W)$ be the flag variety of flags $W_{1} \subset W_{2} \subset \cdots \subset W_{m}$ of linear subspaces of $W$ such that the dimension of $W_{i}$ is $i$. Let $\mathcal{T}^{i}(m, W)$ be the tautological rank $i$ vector bundle on $\mathbf{F}(m, W)$.

Let $V=\mathbf{A}^{\infty}$ be an infinite-dimensional vector bundle over $S$ and set $e=(1,0, \ldots)$. Then $V_{n}$ denotes the $n$-fold product of $V$, and $e_{i}^{n} \in V_{n}$ the vector $(0, \ldots, 0, e, 0, \ldots, 0)$ having $e$ precisely at the $i$-th position. Let $F(m)=\operatorname{colim}_{W} \mathbf{F}(m, W)$ and let $\mathcal{T}^{i}(m)=$ $\operatorname{colim}_{W} \mathcal{T}^{i}(m, W)$, where $W$ runs over all finite-dimensional vector subspaces of $V_{n}$. Thus we have a flag $\mathcal{T}^{1}(m) \subset \mathcal{T}^{2}(m) \subset \cdots \subset \mathcal{T}^{m}(m)$ of vector bundles over $F(m)$. Set $L^{i}(m)=\mathcal{T}^{i}(m) / \mathcal{T}^{i-1}(m)$. It is a line bundle over $F(m)$.

Consider the morphism $p_{m}: F(m) \rightarrow F(m-1)$ which maps a flag $W_{1} \subset W_{2} \subset$ $\cdots \subset W_{m}$ to the flag $W_{1} \subset W_{2} \subset \cdots \subset W_{m-1}$. If $W \subset V_{n}$ is a finite-dimensional vector subspace, then the restriction of $p_{m}: F(m) \rightarrow F(m-1)$ to $\mathbf{F}(m, W)$ is a projective space bundle over $\mathbf{F}(m-1, W)$. Thus there exists a tower of projective space bundles $F(m) \rightarrow F(m-1) \rightarrow \cdots \rightarrow F(1)=\mathbf{P}\left(V_{n}\right)$. The projective bundle theorem implies that

$$
E^{*, *}(F(n))=E^{*, *}(k)\left[\left[t_{1}, t_{2}, \ldots, t_{n}\right]\right]
$$

(the formal power series in $n$ variables), where $t_{i}=c\left(L^{i}(n)\right)$ is the first Chern class of the line bundle $L^{i}(n)$ over $F(n)$.

Consider the morphism $q: F(n) \rightarrow \mathbf{G r}(n)$, which sends the flag

$$
W_{1} \subset W_{2} \subset \cdots \subset W_{n}
$$

to the space $W_{n}$. It can be decomposed as a tower of projective space bundles. In particular, the pull-back map $q^{*}: E^{*, *}(\mathbf{G r}(n)) \rightarrow E^{*, *}(F(n))$ is a monomorphism. It maps the class $c_{i}$ to the symmetric polynomial

$$
\sigma_{i}=t_{1} t_{2} \cdots t_{i}+\cdots+t_{n-i+1} \cdots t_{n-1} t_{n}
$$

So the image of $q^{*}$ contains $E^{*, *}(k)\left[\left[\sigma_{1}, \sigma_{2}, \ldots, \sigma_{n}\right]\right]$. It remains to check that the image of $q^{*}$ is contained in $E^{*, *}(k)\left[\left[\sigma_{1}, \sigma_{2}, \ldots, \sigma_{n}\right]\right]$. To do that consider another variety. 
Namely, let $V^{0}$ be the $n$-dimensional subspace of $V_{n}$ generated by the vectors $e_{i}^{n}$ 's. Let $l_{i}^{n}$ be the line generated by the vector $e_{i}^{n}$. Let $V_{i}^{0}$ be a subspace of $V^{0}$ generated by all $e_{j}^{n}$ 's with $j \leqslant i$. So one has a flag $V_{1}^{0} \subset V_{2}^{0} \subset \cdots \subset V_{n}^{0}=V^{0}$. We denote this flag $F^{0}$. For each vector subspace $W$ in $V_{n}$ containing $V^{0}$ consider three algebraic subgroups of the general linear group $\mathbb{G L}_{W}$. Namely, set

$$
P_{W}=\operatorname{Stab}\left(V^{0}\right), B_{W}=\operatorname{Stab}\left(F^{0}\right), T_{W}=\operatorname{Stab}\left(l_{1}^{n}, l_{2}^{n}, \ldots, l_{n}^{n}\right) .
$$

The group $T_{W}$ stabilizes each line $l_{i}^{n}$. Clearly, $T_{W} \subset B_{W} \subset P_{W}$ and $\operatorname{Gr}(n, W)=$ $\mathbb{G L}_{W} / P_{W}, \mathbf{F}(n, W)=\mathbb{G L}_{W} / B_{W}$ Set $M(n, W)=\mathbb{G L}_{W} / T_{W}$. One has a tower of obvious morphisms

$$
M(n, W) \stackrel{r_{W}}{\longrightarrow} \mathbf{F}(n, W) \stackrel{q_{W}}{\longrightarrow} \mathbf{G r}(n, W) .
$$

Set $M(n)=\operatorname{colim}_{W} M(n, W)$, where $W$ runs over all finite-dimensional subspaces $W$ of $V_{n}$ containing $V^{0}$. Now one has a tower of morphisms

$$
M(n) \stackrel{r}{\rightarrow} F(n) \stackrel{q}{\rightarrow} \mathbf{G r}(n) .
$$

The morphisms $r_{W}$ can be decomposed in a tower of affine space bundles. Hence it induces an isomorphism on any cohomology theory. Choose a family

$$
V_{n}^{0}=W_{0} \subset W_{1} \subset W_{2} \subset \cdots
$$

of finite-dimensional subspaces of $V_{n}$ such that $V_{n}=\cup W_{i}$. Then $F(n)=\cup \mathbf{F}\left(n, W_{i}\right)$ and $M(n)=\cup M\left(n, W_{i}\right)$. The short exact sequence

$$
0 \rightarrow{\underset{i \geqslant 0}{\overleftarrow{i}}}^{1} E^{*-1, *}\left(\mathbf{F}\left(n, W_{i}\right)\right) \rightarrow E^{*, *}(F(n)) \rightarrow \lim _{i \geqslant 0} E^{*, *}\left(\mathbf{F}\left(n, W_{i}\right)\right) \rightarrow 0
$$

as described in [7, Lemma A.34], and a similar sequence for $E^{*}$, -groups of the spaces $M\left(n, W_{i}\right)$, show that the pull-back map

$$
r^{*}: E^{*, *}(F(n)) \rightarrow E^{*, *}(M(n))
$$

is an isomorphism. Permuting vectors $e_{i}^{n}$ 's yields an inclusion $\Sigma_{n} \subset \mathbb{G} \mathbb{L}\left(V^{0}\right)$ of the symmetric group $\Sigma_{n}$ in $\mathbb{G} \mathbb{L}\left(V^{0}\right)$. The action of $\Sigma_{n}$ by the conjugation on $\mathbb{G L}_{W}$ normalizes the subgroups $T_{W}$ and $P_{W}$. Thus $\Sigma_{n}$ acts as on $M(n)$ so on $\operatorname{Gr}(n)$ and the morphism $q \circ r: M(n) \rightarrow \mathbf{G r}(n)$ respects this action. Note that the action of $\Sigma_{n}$ on $\mathbf{G r}(n)$ is trivial and the action of $\Sigma_{n}$ on $E^{*, *}(M(n))$ permutes the variable $t_{1}, t_{2}, \ldots, t_{n}$. Thus the image of $(q \circ r)^{*}$ is contained in $E^{*, *}(S)\left[\left[\sigma_{1}, \sigma_{2}, \ldots, \sigma_{n}\right]\right]$. Whence the same holds for the image of $q^{*}$. The theorem is proven.

The projection from the product $\mathbf{G r}(m) \times \mathbf{G r}(n)$, to the $j$-th factor is called $p_{j}$. For every integer $i \geqslant 0$ set $c_{i}^{\prime}=p_{1}^{*}\left(c_{i}(\mathcal{T}(m))\right)$ and $c_{i}^{\prime \prime}=p_{2}^{*}\left(c_{i}(\mathcal{T}(n))\right)$

Theorem 2.3. Suppose $E$ is an oriented commutative $\mathbf{P}^{1}$-ring spectrum. There is an isomorphism

$$
E^{*, *}((\mathbf{G r}(m) \times \mathbf{G r}(n)))=E^{*, *}(S)\left[\left[c_{1}^{\prime}, c_{2}^{\prime}, \ldots, c_{m}^{\prime}, c_{1}^{\prime \prime}, c_{2}^{\prime \prime}, \ldots, c_{n}^{\prime \prime}\right]\right],
$$

where the right-hand side denotes the formal power series ring on $c_{i}^{\prime}$ and $c_{j}^{\prime \prime}$ with 
coefficients in $E^{*, *}(S)$. The inclusion

$$
i_{m, n}: \mathbf{G r}(m) \times \mathbf{G r}(n) \hookrightarrow \mathbf{G r}(m+1) \times \mathbf{G r}(n+1)
$$

satisfies

$$
i_{m, n}^{*}\left(c_{r}^{\prime}\right)=c_{r}^{\prime} \quad \text { for } \quad r<m+1, i_{m, n}^{*}\left(c_{m+1}^{\prime}\right)=0
$$

and

$$
i_{m, n}^{*}\left(c_{r}^{\prime \prime}\right)=c_{r}^{\prime \prime} \quad \text { for } \quad r<n+1, i_{m, n}^{*}\left(c_{n+1}^{\prime \prime}\right)=0 .
$$

Proof. This follows as in the proof of Theorem 2.2 .

\subsection{The symmetric ring spectrum representing algebraic cobordism}

To give a construction of the symmetric $\mathbf{P}^{1}$-ring spectrum MGL, recall the external product of Thom spaces described in [13, p. 422]. For vector bundles $V \rightarrow X$ and $W \rightarrow Y$ in $\mathcal{S} m / S$, one obtains a canonical map of pointed motivic spaces $\operatorname{Th}(V) \wedge \operatorname{Th}(W) \rightarrow \operatorname{Th}\left(V \times_{S} W\right)$, which is a motivic weak equivalence as defined in [7, Defn. 3.1.6]. In fact, the canonical map becomes an isomorphism after Nisnevich (even Zariski) sheafification.

The algebraic cobordism spectrum appears naturally as a $T$-spectrum, not as a $\mathbf{P}^{1}$-spectrum. Hence we describe it as a symmetric $T$-ring spectrum and obtain a symmetric $\mathbf{P}^{1}$-ring spectrum (and in particular a $\mathbf{P}^{1}$-ring spectrum) by switching the suspension coordinate (see [7, A.6.9]). For $m, n \geqslant 0$, let $\mathcal{T}(n, m n) \rightarrow \mathbf{G r}(n, m n)$ denote the tautological vector bundle over the Grassmann scheme of $n$-dimensional linear subspaces of $\mathbf{A}_{S}^{m n}=\mathbf{A}_{S}^{m} \times_{S} \cdots \times_{S} \mathbf{A}_{S}^{m}$. Permuting the copies of $\mathbf{A}_{S}^{m}$ induces a $\Sigma_{n}$-action on $\mathcal{T}(n, m n)$ and $\mathbf{G r}(n, m n)$ such that the bundle projection is equivariant. The closed embedding $\mathbf{A}_{S}^{m}=\mathbf{A}_{S}^{m} \times\{0\} \hookrightarrow \mathbf{A}_{S}^{m+1}$ defines a closed $\Sigma_{n}$-equivariant embedding $\mathbf{G r}(n, m n) \hookrightarrow \mathbf{G r}(n,(m+1) n)$. In particular, $\mathbf{G r}(n, m n)$ is pointed by $g_{n}: S=\mathbf{G r}(n, n) \hookrightarrow \mathbf{G r}(n, m n)$. The fiber of $\mathbf{G r}(n, m n)$ over $g_{n}$ is $\mathbf{A}_{S}^{n}$. Let $\mathbf{G r}(n)$ be the colimit of the sequence

$$
\mathbf{G r}(n, n) \hookrightarrow \mathbf{G r}(n, 2 n) \hookrightarrow \cdots \hookrightarrow \mathbf{G r}(n, m n) \hookrightarrow \cdots
$$

in the category of pointed motivic spaces over $S$. The pullback diagram

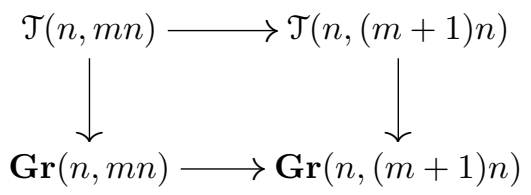

induces a $\Sigma_{n}$-equivariant inclusion of Thom spaces

$$
\operatorname{Th}(\mathcal{T}(n, m n)) \hookrightarrow \operatorname{Th}(\mathcal{T}(n,(m+1) n)) .
$$

Let $\mathbb{M} \mathbb{G}_{n}$ denote the colimit of the resulting sequence

$$
\mathbb{M} \mathbb{G} \mathbb{L}_{n}=\operatorname{colim}_{m \geqslant n} \operatorname{Th}(\mathcal{T}(n, m n))
$$

with the induced $\Sigma_{n}$-action. There is a closed embedding

$$
\mathbf{G r}(n, m n) \times \mathbf{G r}(p, m p) \hookrightarrow \mathbf{G r}(n+p, m(n+p)),
$$


which sends the linear subspaces $V \hookrightarrow \mathbf{A}^{m n}$ and $W \hookrightarrow \mathbf{A}^{m p}$ to the product subspace $V \times W \hookrightarrow \mathbf{A}^{m n} \times \mathbf{A}^{m p}=\mathbf{A}^{m(n+p)}$. In particular, $\left(g_{n}, g_{p}\right)$ maps to $g_{n+p}$. The inclusion (6) is covered by a map of tautological vector bundles and thus gives a canonical map of Thom spaces

$$
\operatorname{Th}(\mathcal{T}(n, m n)) \wedge \operatorname{Th}(\mathcal{T}(p, m p)) \rightarrow \operatorname{Th}(\mathcal{T}(n+p, m(n+p))),
$$

which is compatible with the colimit (5). Furthermore, the map (7) is $\Sigma_{n} \times \Sigma_{p^{-}}$ equivariant, where the product acts on the target via the standard inclusion $\Sigma_{n} \times \Sigma_{p} \subseteq \Sigma_{n+p}$. After taking colimits, the result is a $\Sigma_{n} \times \Sigma_{p}$-equivariant map

$$
\mu_{n, p}: \mathbb{M} \mathbb{G}_{n} \wedge \mathbb{M} \mathbb{G}_{p} \rightarrow \mathbb{M} \mathbb{G} \mathbb{L}_{n+p}
$$

of pointed motivic spaces (see [13, p. 422]). The inclusion of the fiber $\mathbf{A}^{p}$ over $g_{p}$ in $\mathcal{T}(p)$ induces an inclusion $\operatorname{Th}\left(\mathbb{A}^{p}\right) \subset \operatorname{Th}(\mathcal{T}(p))=\mathbb{M} \mathbb{G}_{p}$. Precomposing it with the canonical $\Sigma_{p}$-equivariant map of pointed motivic spaces,

$$
\operatorname{Th}\left(\mathbb{A}^{1}\right) \wedge \operatorname{Th}\left(\mathbb{A}^{1}\right) \wedge \cdots \wedge \operatorname{Th}\left(\mathbb{A}^{1}\right) \rightarrow \operatorname{Th}\left(\mathbb{A}^{p}\right)
$$

defines a family of maps $e_{p}:\left(\Sigma_{T}^{\infty} S_{+}\right)_{p}=T^{\wedge p} \rightarrow \mathbb{M} \mathbb{G L}_{p}$. Inserting it in the inclusion (8) yields $\Sigma_{n} \times \Sigma_{p}$-equivariant structure maps

$$
\mathbb{M G} \mathbb{L}_{n} \wedge \operatorname{Th}\left(\mathbb{A}^{1}\right) \wedge \operatorname{Th}\left(\mathbb{A}^{1}\right) \wedge \cdots \wedge \operatorname{Th}\left(\mathbb{A}^{1}\right) \rightarrow \mathbb{M} \mathbb{G L}_{n+p}
$$

of the symmetric $T$-spectrum $\mathbb{M} \mathbb{G} L$. The family of $\Sigma_{n} \times \Sigma_{p}$-equivariant maps (8) form a commutative, associative and unital multiplication on the symmetric $T$-spectrum $\mathbb{M G L}$ (see [3, Sect. 4.3]). Regarded as a T-spectrum it coincides with Voevodsky's spectrum MGL described in [13, 6.3].

Let $\bar{T}$ be the Nisnevich sheaf associated to the presheaf $X \mapsto \mathbf{P}^{1}(X) /\left(\mathbf{P}^{1}-\{0\}\right)(X)$ on the Nisnevich site $\mathcal{S} m / S$. The canonical covering of $\mathbf{P}^{1}$ supplies an isomorphism

$$
T=\operatorname{Th}\left(\mathbf{A}_{S}^{1}\right) \stackrel{\cong}{T}
$$

of pointed motivic spaces. This isomorphism induces an isomorphism $\mathbf{M S S}_{T}(S) \cong$ $\mathbf{M S S}_{\bar{T}}(S)$ of the categories of symmetric $T$-spectra and symmetric $\bar{T}$-spectra. In particular, $\mathbb{M} \mathbb{G} \mathbb{L}$ may be regarded as a symmetric $\bar{T}$-spectrum by just changing the structure maps up to an isomorphism. Note that the isomorphism of categories respects both the symmetric monoidal structure and the model structure. The canonical projection $p: \mathbf{P}^{1} \rightarrow \bar{T}$ is a motivic weak equivalence, because $\mathbf{A}^{1}$ is contractible. It induces a Quillen equivalence

$$
\operatorname{MSS}(S)=\operatorname{MSS}_{\mathbf{P}^{1}}(S) \underset{p^{*}}{\stackrel{p_{\sharp}}{\rightleftarrows}} \operatorname{MSS}_{\bar{T}}(S)
$$

when equipped with model structures as described in [3] (see [7, A.6.9]). The right adjoint $p^{*}$ is very simple: it sends a symmetric $\bar{T}$-spectrum $E$ to the symmetric $\mathbf{P}^{1}$ spectrum having terms $\left(p^{*}(E)\right)_{n}=E_{n}$ and structure maps

$$
E_{n} \wedge \mathbf{P}^{1} \stackrel{E_{n} \wedge p}{\longrightarrow} E \wedge \bar{T} \stackrel{\text { structure map }}{\longrightarrow} E_{n+1}
$$

In particular MGL $:=p^{*} \mathbb{M} \mathbb{G} \mathbb{L}$ is a symmetric $\mathbf{P}^{1}$-spectrum by just changing the structure maps. Since $p^{*}$ is a lax symmetric monoidal functor, MGL is a commutative 
monoid in a canonical way. Finally, the identity is a left Quillen equivalence from the model category $\mathbf{M S S}^{\mathrm{cm}}(S)$ used in [7] to Jardine's model structure by the proof of $\left[\mathbf{7}\right.$, A.6.4]. Let $\gamma: \operatorname{Ho}\left(\mathbf{M S S}^{\mathrm{cm}}(S)\right) \rightarrow \mathrm{SH}(S)$ denote the equivalence obtained by regarding a symmetric $\mathbf{P}^{1}$-spectrum just as a $\mathbf{P}^{1}$-spectrum.

Definition 2.4. Let (MGL, $\mu_{\mathrm{MGL}}, e_{\mathrm{MGL}}$ ) denote the commutative $\mathbf{P}^{1}$-ring spectrum, which is the image $\gamma(\mathrm{MGL})$ of the commutative symmetric $\mathbf{P}^{1}$-ring spectrum MGL in the motivic stable homotopy category $\mathrm{SH}(S)$.

\subsection{Cohomology of the algebraic cobordism spectrum}

Let $(E, t h)$ be an oriented commutative $\mathbf{P}^{1}$-ring spectrum and let $V \mapsto t h(V)$ be the Thom classes theory given by equation (3). We will compute $E^{*, *}$ (MGL) and $E^{*, *}(\mathrm{MGL} \wedge \mathrm{MGL})$ in this short section.

By [7, Cor. 2.1.4], the group $E^{*, *}(\mathrm{MGL})$ fits into the short exact sequence

$$
0 \rightarrow \lim ^{1} E^{*+2 i-1, *+i}(\operatorname{Th}(\mathcal{T}(i))) \rightarrow E^{*, *}(\mathrm{MGL}) \rightarrow \underset{\longleftarrow}{\lim } E^{*+2 i, *+i}(\operatorname{Th}(\mathcal{T}(i))) \rightarrow 0,
$$

where the connecting maps in the tower are given by the top line of the commutative diagram

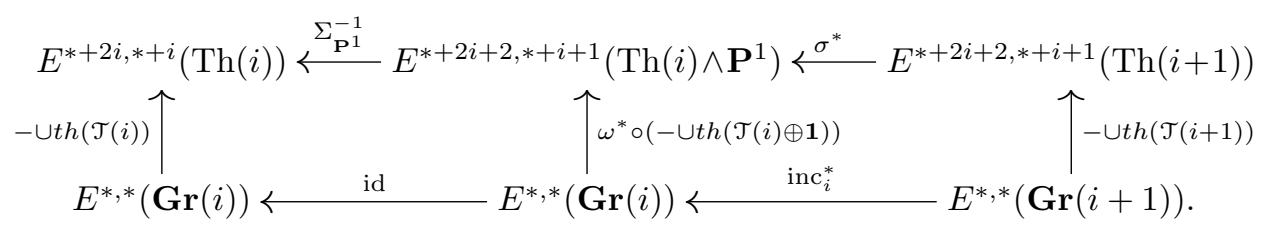

Here $\omega: \operatorname{Th}(V) \wedge \mathbf{P}^{1} \rightarrow T h(V \oplus \mathbf{1})$ is the canonical map described in Corollary 1.6 and $\sigma: \mathrm{Th}_{i} \wedge \mathbf{P}^{1} \rightarrow \mathrm{Th}_{i+1}$ is the structure map of the $\mathbf{P}^{1}$-spectrum MGL. The pullbacks inc $_{i}^{*}$ are all surjective by Theorem 1.5. So we proved the following

Lemma 2.5. The canonical map

$$
E^{*, *}(\mathrm{MGL}) \rightarrow \lim _{\longleftarrow} E^{*+2 i, *+i}(\operatorname{Th}(\mathcal{T}(i)))=E^{*, *}(S)\left[\left[c_{1}, c_{2}, c_{3}, \ldots\right]\right]
$$

is an isomorphism of two-sided $E^{*, *}(S)$-modules.

To compute $E^{*, *}(\mathrm{MGL} \wedge \mathrm{MGL})$, recall that the group $E^{*, *}(\mathrm{MGL} \wedge \mathrm{MGL})$ fits into the short exact sequence

$$
\begin{aligned}
& 0 \rightarrow \lim ^{1} E^{*+4 i-1, *+2 i}(\operatorname{Th}(\mathcal{T}(i)) \wedge \operatorname{Th}(\mathcal{T}(i))) \rightarrow E^{*, *}(\mathrm{MGL} \wedge \mathrm{MGL}) \\
& \rightarrow \underset{\lim }{\longleftarrow} E^{*+4 i, *+2 i}(\mathrm{Th}(\mathcal{T}(i)) \wedge \operatorname{Th}(\mathcal{T}(i))) \rightarrow 0
\end{aligned}
$$

by [7, Cor. 2.1.5]. Note that since $\operatorname{Th}(\mathcal{T}(i)) \wedge \operatorname{Th}(\mathcal{T}(i)) \cong \operatorname{Th}(\mathcal{T}(i) \times \mathcal{T}(i))$, there is a Thom isomorphism $E^{*+4 i-1, *+2 i}(\operatorname{Th}(\mathcal{T}(i) \times \mathcal{T}(i))) \cong E^{*-1, *}(\mathbf{G r}(i) \times \mathbf{G r}(i))$ by Theorem 1.5. The $\lim ^{1}$-group is trivial because the connecting maps coincide with the pull-back maps

$$
E^{*-1, *}(\mathbf{G r}(i+1) \times \mathbf{G r}(i+1)) \rightarrow E^{*-1, *}(\mathbf{G r}(i) \times \mathbf{G r}(i))
$$

and these are surjective by Theorem 2.3. This implies the following 
Lemma 2.6. The canonical map

$$
\begin{aligned}
E^{*, *}(\mathrm{MGL} \wedge \mathrm{MGL}) \rightarrow \lim E^{*+4 i, *+2 i}(\operatorname{Th}(\mathcal{T}(i)) \wedge & \operatorname{Th}(\mathcal{T}(i))) \\
& =E^{*, *}(S)\left[\left[c_{1}^{\prime}, c_{1}^{\prime \prime}, c_{2}^{\prime}, c_{2}^{\prime \prime}, \ldots\right]\right]
\end{aligned}
$$

is an isomorphism of $E^{*, *}(S)$-modules. Here $c_{i}^{\prime}$ is the $i$-th Chern class coming from the first factor of $\mathbf{G r} \times \mathbf{G r}$ and $c_{i}^{\prime \prime}$ is the $i$-th Chern class coming from the second factor.

\subsection{A universality theorem for the algebraic cobordism spectrum}

The complex cobordism spectrum, equipped with its natural orientation, is a universal oriented ring cohomology theory by Quillen's universality theorem [10]. In this section we prove a motivic version of Quillen's universality theorem. Over a field, the statement is contained already in [12]. Recall that the $\mathbf{P}^{1}$-ring spectrum MGL carries a canonical orientation $t h^{\mathrm{MGL}}$ as defined in Example 1.4. It is the canonical map

$$
t h^{\mathrm{MGL}}: \Sigma_{\mathbf{P}^{1}}^{\infty} \operatorname{Th}(\mathcal{T}(1))(-1) \rightarrow \mathrm{MGL}
$$

of $\mathbf{P}^{1}$-spectra.

Theorem 2.7 (Universality Theorem). Let $E$ be a commutative $\mathbf{P}^{1}$-ring spectrum. The assignment

$$
\varphi \mapsto \varphi\left(t h^{\mathrm{MGL}}\right) \in E^{2,1}(\operatorname{Th}(\mathcal{T}(1)))
$$

identifies the set of homomorphisms

$$
\varphi: \mathrm{MGL} \rightarrow E
$$

of $\mathbf{P}^{1}$-ring spectra in the motivic stable homotopy category $\mathrm{SH}(S)$ with the set of orientations of $E$. The inverse bijection sends an orientation th $\in E^{2,1}(\operatorname{Th}(\mathcal{T}(1)))$ to the unique morphism

$$
\varphi \in E^{0,0}(\mathrm{MGL})=\operatorname{Hom}_{\mathrm{SH}(S)}(\mathrm{MGL}, E)
$$

such that $u_{i}^{*}(\varphi)=\operatorname{th}(\mathcal{T}(i)) \in E^{2 i, i}(\operatorname{Th}(\mathcal{T}(i)))$, where $\operatorname{th}(\mathcal{T}(i))$ is given by (3) and $u_{i}: \Sigma_{\mathbf{P}^{1}}^{\infty} \operatorname{Th}(\mathcal{T}(i))(-i) \rightarrow$ MGL is the canonical map of $\mathbf{P}^{1}$-spectra.

Proof. Let $\varphi$ : MGL $\rightarrow E$ be a homomorphism of monoids in $\operatorname{SH}(S)$. The class $t h:=\varphi\left(t h^{\mathrm{MGL}}\right)$ is an orientation of $E$, because

$$
\left.\varphi(t h)\right|_{T h(\mathbf{1})}=\varphi\left(\left.t h\right|_{T h(\mathbf{1})}\right)=\varphi\left(\Sigma_{\mathbf{P}^{1}}(1)\right)=\Sigma_{\mathbf{P}^{1}}(\varphi(1))=\Sigma_{\mathbf{P}^{1}}(1) .
$$

Now suppose $t h^{E} \in E^{2 i, i}(\operatorname{Th}(\mathcal{O}(-1)))$ is an orientation of $E$. Let $V \mapsto t h(V)$ be the Thom classes theory given by equation (3). We will construct a monoid homomorphism $\varphi:$ MGL $\rightarrow E$ in $\operatorname{SH}(S)$ such that $u_{i}^{*}(\varphi)=t h(\mathcal{T}(i))$ and prove its uniqueness. To do so recall that the canonical map $E^{*, *}(\mathrm{MGL}) \rightarrow \lim E^{*+2 i, *+i}(\operatorname{Th}(\mathcal{T}(i)))$ is an isomorphism by Lemma 2.5. The connecting maps in the tower are given by the top line of diagram (10). The family of elements $t h(\mathcal{T}(i))$ is an element in the lim-group because diagram (10) commutes. Thus there is a unique element $\varphi \in E^{0,0}(\mathrm{MGL})$ with $u_{i}^{*}(\varphi)=\operatorname{th}(\mathcal{T}(i))$. 
We claim that $\varphi$ is a monoid homomorphism. To check that it respects the multiplicative structure, consider the diagram

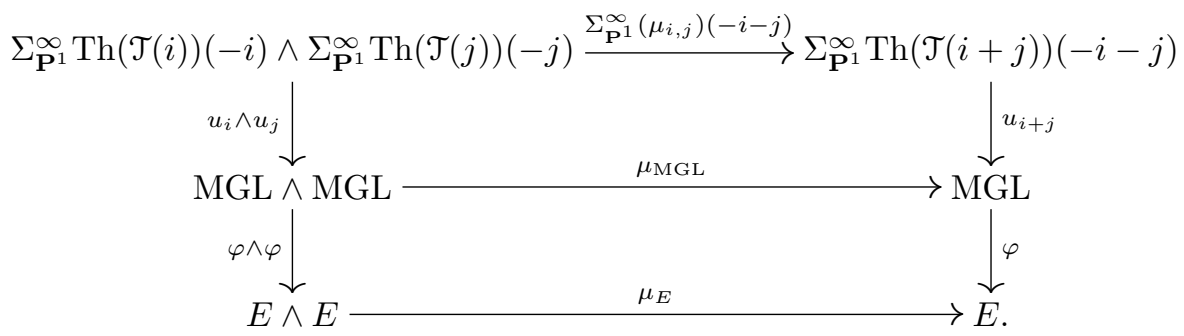

Its enveloping square commutes in $\mathrm{SH}(S)$ by the chain of relations

$$
\begin{aligned}
\varphi \circ u_{i+j} \circ \Sigma_{\mathbf{P}^{1}}^{\infty}\left(\mu_{i, j}\right)(-i-j) & =\mu_{i, j}^{*}(\operatorname{th}(\mathcal{T}(i+j)))=\operatorname{th}\left(\mu_{i, j}^{*}(\mathcal{T}(i+j))\right)=\operatorname{th}(\mathcal{T}(i) \times \mathcal{T}(j)) \\
& =\operatorname{th}(\mathcal{T}(i)) \times \operatorname{th}(\mathcal{T}(j))=\mu_{E}(\operatorname{th}(\mathcal{T}(i)) \wedge \operatorname{th}(\mathcal{T}(j))) \\
& =\mu_{E} \circ\left(\left(\varphi \circ u_{i}\right) \wedge\left(\varphi \circ u_{j}\right)\right) .
\end{aligned}
$$

The canonical map $E^{*, *}(\mathrm{MGL} \wedge \mathrm{MGL}) \rightarrow \underset{\longleftarrow}{\lim } E^{*+4 i, *+2 i}(\operatorname{Th}(\mathcal{T}(i)) \wedge \operatorname{Th}(\mathcal{T}(i)))$ is an isomorphism by Lemma 2.6. Now the equality

$$
\varphi \circ u_{i+i} \circ \Sigma_{\mathbf{P}^{1}}^{\infty}\left(\mu_{i, i}\right)(-2 i)=\mu_{E} \circ\left(\left(\varphi \circ u_{i}\right) \wedge\left(\varphi \circ u_{i}\right)\right)
$$

shows that $\mu_{E} \circ(\varphi \wedge \varphi)=\varphi \circ \mu_{\mathrm{MGL}}$ in $\mathrm{SH}(S)$.

To prove the theorem it remains to check that the two assignments described in the theorem are inverse to each other. An orientation $t h \in E^{2,1}(\operatorname{Th}(\mathcal{O}(-1)))$ induces a morphism $\varphi$ such that for each $i$ one has $\varphi \circ u_{i}=\operatorname{th}\left(\mathcal{T}_{i}\right)$. The new orientation $t h^{\prime}:=\varphi\left(t h^{\mathrm{MGL}}\right)$ coincides with the original one, because of the chain of relations

$$
t h^{\prime}=\varphi\left(t h^{\mathrm{MGL}}\right)=\varphi\left(u_{1}\right)=\varphi \circ u_{1}=t h(\mathcal{T}(1))=t h(\mathcal{O}(-1))=t h .
$$

On the other hand a homomorphism $\varphi$ of $\mathbf{P}^{1}$-ring spectra defines an orientation $t h:=\varphi\left(t h^{\mathrm{MGL}}\right)$ of $E$. The monoid homomorphism $\varphi^{\prime}$ we obtain then satisfies $u_{i}^{*}\left(\varphi^{\prime}\right)=$ $t h(\mathcal{T}(i))$ for every $i \geqslant 0$. To check that $\varphi^{\prime}=\varphi$, recall that MGL is oriented, so we may use Lemma 2.5 with $E=$ MGL to deduce an isomorphism

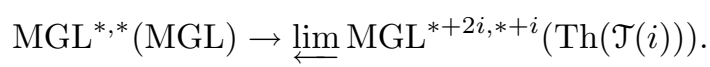

This isomorphism shows that the identity $\varphi^{\prime}=\varphi$ will follow from the identities $u_{i}^{*}\left(\varphi^{\prime}\right)=u_{i}^{*}(\varphi)$ for every $i \geqslant 0$. Since $u_{i}^{*}\left(\varphi^{\prime}\right)=t h\left(\mathcal{T}_{i}\right)$ it remains to check the relation $u_{i}^{*}(\varphi)=\operatorname{th}(\mathcal{T}(i))$. It follows from the

Lemma 2.8. There is an equality $u_{i}=t h^{\mathrm{MGL}}\left(\mathcal{T}(i) \in \operatorname{MGL}^{2 i, i}(\operatorname{Th}(\mathcal{T}(i)))\right.$.

In fact, $u_{i}^{*}(\varphi)=\varphi \circ u_{i}=\varphi\left(u_{i}\right)=\varphi\left(t h^{\mathrm{MGL}}(\mathcal{T}(i))\right)=t h(\mathcal{T}(i))$. The last equality in this chain of relations holds, because $\varphi$ is a monoid homomorphism sending $t h^{\text {MGL }}$ to $t h$. It remains to prove Lemma 2.8. We will do this in the case $i=2$. The general case can be proved similarly. The commutative diagram

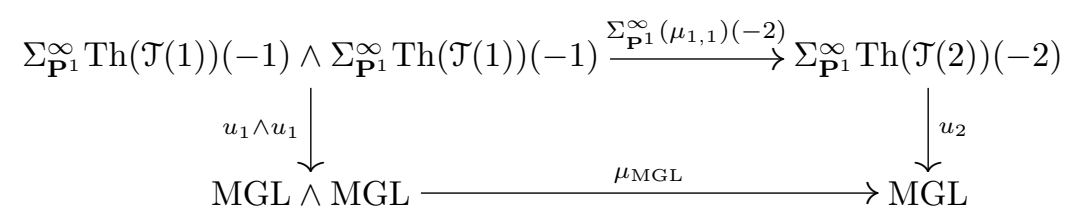


in $\mathrm{SH}(k)$ implies that

$$
\mu_{1,1}^{*}\left(u_{2}\right)=u_{1} \times u_{1} \in \operatorname{MGL}^{4,2}(\operatorname{Th}(\mathcal{T}(1)) \wedge \operatorname{Th}(\mathcal{T}(1)))=\operatorname{MGL}^{4,2}(\operatorname{Th}(\mathcal{T}(1) \times \mathcal{T}(1))) .
$$

The equalities

$$
\begin{aligned}
\mu_{1,1}^{*}\left(t h^{\mathrm{MGL}}(\mathcal{T}(2))\right) & =t h^{\mathrm{MGL}}\left(\mu_{1,1}^{*}(\mathcal{T}(2))\right)=t h^{\mathrm{MGL}}(\mathcal{T}(1) \times \mathcal{T}(1)) \\
& =t h^{\mathrm{MGL}}(\mathcal{T}(1)) \times t h^{\mathrm{MGL}}(\mathcal{T}(1))
\end{aligned}
$$

imply that it remains to prove the injectivity of the map $\mu_{1,1}^{*}$. Consider the commutative diagram

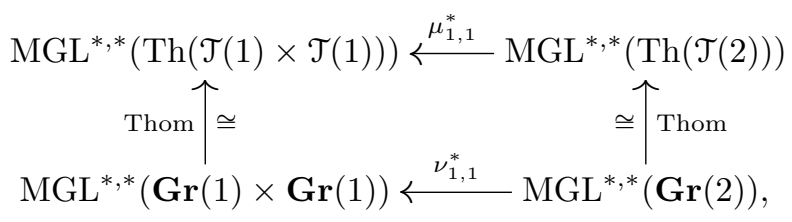

where the vertical arrows are the Thom isomorphisms from Theorem 1.5 and $\nu_{1,1}: \mathbf{G r}(1) \times \mathbf{G r}(1) \hookrightarrow \mathbf{G r}(2)$ is the embedding described by equation (6). For an oriented commutative $\mathbf{P}^{1}$-ring spectrum $(E, t h)$, one has $E^{*, *}(\mathbf{G r}(2))=E^{*, *}(S)\left[\left[c_{1}, c_{2}\right]\right]$ (the formal power series on $c_{1}, c_{2}$ ) by Theorem 2.2. On the other hand,

$$
E^{*, *}(\mathbf{G r}(1) \times \mathbf{G r}(1))=E^{*, *}(S)\left[\left[t_{1}, t_{2}\right]\right]
$$

(the formal power series on $t_{1}, t_{2}$ ) by Theorem 2.3 and the map $\nu_{1,1}^{*}$ sends $c_{1}$ to $t_{1}+t_{2}$ and $c_{2}$ to $t_{1} t_{2}$. Whence $\nu_{1,1}^{*}$ is injective. The proofs of Lemma 2.8 and of Theorem 2.7 are complete.

\section{Universality of MGL and formal group laws}

In this section the universal property of the $\mathbf{P}^{1}$-spectrum MGL will be described in terms of formal group laws. Fix a commutative $\mathbf{P}^{1}$-ring spectrum $E$ and a homomorphism $\varphi$ : MGL $\rightarrow E$ of $\mathbf{P}^{1}$-ring spectra over $S$. Let $z$ be the zero section of the line bundle $\mathcal{O}(-1)$ over $\mathbf{P}^{\infty}$. Then $c^{\mathrm{MGL}}=z^{*}\left(t h^{\mathrm{MGL}}\right)$ is a Chern orientation of MGL and $c=\varphi\left(c^{\mathrm{MGL}}\right)$ is a Chern orientation of $E$. The Chern orientation $c$ defines in the standard way a formal group law $F$ over the commutative ring $E^{2 *, *}(S)$ (see for instance [6, Defn. 3.39] and set $F:=F^{-}$, where $F^{-}$is the formal group law corresponding to the class $c(\mathcal{O}(-1)))$.

If $\varphi_{\text {new }}:$ MGL $\rightarrow E$ is another homomorphism of $\mathbf{P}^{1}$-ring spectra, then the element $c_{\text {new }}:=\varphi_{\text {new }}\left(c^{\mathrm{MGL}}\right) \in E^{2,1}\left(\mathbf{P}^{\infty}\right)$ defines another formal group law $F_{\text {new }}$. Moreover it defines a unique formal power series $\Phi(t) \in E^{2 *, *}(S)$ such that $c_{\text {new }}=\Phi(c)$. It is straightforward to check that $\Phi(t)$ is of the form $t+b_{1} t^{2}+b_{2} t^{3}+\cdots$ with $b_{i} \in E^{-2 i,-i}(S)$ and $\Phi\left(F\left(t_{1}, t_{2}\right)\right)=F_{\text {new }}\left(\Phi\left(t_{1}\right), \Phi\left(t_{2}\right)\right)$. In other words, $\Phi(t)$ is an isomorphism $F \rightarrow F_{\text {new }}$ of formal group laws.

Theorem 3.1. Let $(E, c)$ be an oriented commutative $\mathbf{P}^{1}$-ring spectrum over $S$. The assignment $\varphi_{\text {new }} \mapsto\left(F_{\text {new }}, \Phi(t)\right)$ is a bijection from the set of all homomorphisms $\mathrm{MGL} \rightarrow E$ of $\mathbf{P}^{1}$-ring spectra in $\mathrm{SH}(S)$ to the set of all pairs $\left(F^{\prime}\left(t_{1}, t_{2}\right), \Psi(t)\right)$, where $F^{\prime}$ is a formal group law over the ring $E^{2 * *}(S)$ and $\Psi(t): F\left(t_{1}, t_{2}\right) \rightarrow F^{\prime}\left(t_{1}, t_{2}\right)$ is an isomorphism of formal group laws as above. 
Proof. Consider the set of all formal power series $\Psi(t) \in E^{2 *, *}(S)[[t]]$ of the form described above. This set forms a group under the substitution of the power series: $\left(\Psi_{2} \circ \Psi_{1}\right)(t):=\Phi_{2}\left(\Psi_{1}(t)\right)$. The series $t$ is the unit of this group. For a series $\Psi$ in this group we will write $\Psi^{-1}$ for its inverse.

By straightforward calculation one may check that the assignments

$$
\left(F^{\prime}\left(t_{1}, t_{2}\right), \Psi(t)\right) \mapsto \Psi(t) \text { and } \Psi(t) \mapsto\left(\Psi\left(F\left(\Psi^{-1}\left(t_{1}\right), \Psi^{-1}\left(t_{2}\right)\right)\right), \Psi(t)\right)
$$

are mutually inverse bijections of the set of all pairs from the theorem with the set of all formal power series $\Psi(t) \in E^{2 *, *}(S)[[t]]$ such that $\Psi(t)=t+b_{1} t^{2}+b_{2} t^{3}+\cdots$, with $b_{i} \in E^{-2 i,-i}(S)$ for all $i$. Secondly, note that the set of all formal power series $\Psi(t) \in E^{2 *, *}(S)[[t]]$ such that $\Psi(t)=t+b_{1} t^{2}+b_{2} t^{3}+\cdots$ with $b_{i} \in E^{-2 i,-i}(S)$ is in a bijective correspondence with the set of all Chern orientations $c^{\prime} \in E^{2,1}\left(\mathbf{P}^{\infty}\right)$ of $E$. Namely, a formal power series $\Psi(t)$ as above maps to the Chern orientation $\Psi(c) \in$ $E^{2,1}\left(\mathbf{P}^{\infty}\right)$. Given a Chern orientation $c^{\prime}$ of $E$, let $\Psi(t) \in E^{2 *, *}(S)[[t]]$ be the unique formal power series such that $c^{\prime}=\Psi(c)$. This supplies two mutually inverse bijections.

To prove the theorem it remains to check that the assignment $\varphi_{\text {new }} \mapsto \varphi_{\text {new }}\left(c^{\mathrm{MGL}}\right)$ is a bijection of the set of all homomorphisms MGL $\rightarrow E$ of $\mathbf{P}^{1}$-ring spectra with the set of all Chern orientations of $E$.

To do that, recall that the assignment $\varphi_{\text {new }} \mapsto \varphi_{\text {new }}\left(t h^{\mathrm{MGL}}\right)$ is a bijection of the set of all ring morphisms $\varphi_{\text {new }}$ : MGL $\rightarrow E$ with the set of all Thom orientations of $E$ (see Theorem 2.7). As well the set of Thom orientations of $E$ is in in bijection with the set of Chern orientations via the assignment $t h \mapsto z^{*}(t h)$ (see [6, Thm. 3.5]). Clearly $z^{*}\left(\varphi_{\text {new }}\left(t h^{\mathrm{MGL}}\right)\right)=\varphi_{\text {new }}\left(c^{\mathrm{MGL}}\right)$. Thus the assignment $\varphi_{\text {new }} \mapsto \varphi_{\text {new }}\left(c^{\mathrm{MGL}}\right)$ is indeed a bijection, which completes the proof.

Remark 3.2. The bijection inverse to $\varphi_{\text {new }} \mapsto\left(F_{\text {new }}, \Phi(t)\right)$ is given as follows. Take $c_{\text {new }}:=\Phi(c)$, construct a Thom classes theory using formulas (2) and (3), and let $\varphi:$ MGL $\rightarrow E$ be the unique homomorphism of $\mathbf{P}^{1}$-ring spectra such that for every

$n$ the composition $\Sigma_{\mathbf{P}^{1}}^{\infty} \operatorname{Th}(\mathcal{T}(n))(-n) \stackrel{u_{n}}{\longrightarrow}$ MGL $\stackrel{\varphi}{\longrightarrow} E$ coincides with the Thom class $\operatorname{th}(\mathcal{T}(n))$ of the bundle $\mathcal{T}(n)$ (here $u_{n}$ is the canonical morphism from Theorem 2.7).

\section{Acknowledgements}

The first author thanks the SFB-701 at the Universität Bielefeld, the RTN-Network HPRN-CT-2002-00287, the RFFI-grant 03-01-00633a, and INTAS-05-1000008-8118 for their support. The third author thanks the Fields Institute for Research in Mathematical Sciences for its support during the Thematic Program on Geometric Applications of Homotopy Theory.

\section{References}

[1] D. Gepner and V. Snaith, On the motivic spectra representing algebraic cobordism and algebraic $K$-theory, preprint; arXiv:0712.2817v2 [math.AG].

[2] J. Hornbostel and S. Yagunov, Rigidity for Henselian local rings and $\mathbf{A}^{1}$ representable theories, Math. Z 255 (2007), no. 2, 437-449.

[3] J.F. Jardine, Motivic symmetric spectra, Doc. Math. 5 (2000), 445-553. 
[4] F. Morel, On the motivic $\pi_{0}$ of the sphere spectrum, in Axiomatic, enriched and motivic homotopy theory, NATO Sci. Ser. II Math. Phys. Chem. 131, 219-260, Kluwer Acad. Publ., Dordrecht, 2004.

[5] F. Morel and V. Voevodsky, $\mathbf{A}^{1}$-homotopy theory of schemes, Publ. Math. IHES 90 (1999), 45-143.

[6] I. Panin, Oriented cohomology theories of algebraic varieties, Special issue in honor of Hyman Bass on his seventieth birthday. Part III, K-Theory 30 (2003), no. 3, 265-314.

[7] I. Panin, K. Pimenov, and O. Röndigs, On Voevodsky's algebraic $K$-theory spectrum BGL, The Abel symposium 2007 (Oslo, Norway, Aug. 5-10, 2007), to appear, Springer-Verlag, New York; arXiv:0709.3905v1 [math.AG].

[8] I. Panin, K. Pimenov, and O. Röndigs, On the relation of Voevodsky's algebraic cobordism to Quillen's K-theory, Invent. Math. (2008), to appear; arXiv:0709.4124v1 [math.AG].

[9] I. Panin and S. Yagunov, Rigidity for orientable functors, J. Pure and Appl. Algebra 172 (2002), no. 1, 49-77.

[10] D. Quillen, On the formal group laws of unoriented and complex cobordism theory, Bull. Amer. Math. Soc. 75 (1969), 1293-1298.

[11] M. Spitzweck and P.A. Østvær, The Bott inverted infinite projective space is $K$-theory, preprint (2007); http://www.math.uio.no/ paularne/bott.pdf.

[12] G. Vezzosi, Brown-Peterson spectra in stable $\mathbf{A}^{1}$-homotopy theory, Rend. Semin. Mat. Univ. Padova 106 (2001), 47-64.

[13] V. Voevodsky, $\mathbf{A}^{1}$-homotopy theory, Doc. Math. 1998, Extra Vol. ICM I (Berlin, 1998), 579-604.

Ivan Panin paniniv@gmail.com

Universität Bielefeld, SFB 701, Bielefeld, Germany, and Steklov Institute of Mathematics at St. Petersburg, Russia

Konstantin Pimenov kip302002@yahoo.com

Steklov Institute of Mathematics at St. Petersburg, Russia

Oliver Röndigs oroendig@math.uni-osnabrueck.de

Institut für Mathematik, Universität Osnabrück, Osnabrück, Germany 